Preprint typeset in JHEP style - PAPER VERSION

DFTT $15 / 2010$

\title{
Vector-Vector scattering at the LHC with two charged leptons and two neutrinos in the final state.
}

\author{
Alessandro Ballestrero $^{a}$, Diogo Buarque Franzosi ${ }^{a, b}$ and Ezio Maina ${ }^{a, b}$ \\ ${ }^{a}$ INFN, Sezione di Torino, Italy, \\ Via Giuria 1, 10125 Torino, Italy, \\ ${ }^{b}$ Dipartimento di Fisica Teorica, Università di Torino, Italy \\ Via Giuria 1, 10125 Torino, Italy. \\ Email: ballestrero@to.infn.it, buarque@to.infn.it, maina@to.infn.it.
}

\begin{abstract}
A complete parton level analysis of $2 \ell 2 \nu 2 j$ and $4 \ell 2 j, \ell=\mu, e$ production at the LHC is presented, including all processes at order $\mathcal{O}\left(\alpha_{E M}^{6}\right), \mathcal{O}\left(\alpha_{E M}^{4} \alpha_{S}^{2}\right)$. The infinite Higgs mass scenario, which is considered as a benchmark for strong scattering theories and is the limiting case for composite Higgs models, and one example of Strongly Interacting Light Higgs models are confronted with the Standard Model light Higgs predictions. This analysis is combined with the results in the $\ell \nu+$ four jets, the $\ell^{-} \ell^{+}+$four jets and the $3 \ell \nu+$ two jets channels presented in previous papers, in order to determine whether these alternative Higgs frameworks can be detected as an excess of events in boson-boson scattering.
\end{abstract}




\section{Contents}

1. Introduction 1

2. Calculation 3

3. The $2 j \ell^{ \pm} \ell^{\prime \pm} \nu \nu$ channel: two same-sign leptons in the final state $\quad 7$

4. The $2 j Z Z \rightarrow 2 j \ell^{+} \ell^{-} \nu \bar{\nu}$ channel: two opposite-sign same-flavour leptons in the final state

5. The $2 j W^{+} W^{-} \rightarrow 2 j \ell^{+} \ell^{\prime-} \nu \bar{\nu}$ channel: two opposite-sign leptons in the final state

6. The $2 j 4 \ell$ channel

\section{Introduction}

Whether or not the search for a light Higgs boson at the LHC will be successful, vector boson scattering processes will require careful analysis. In fact, the corresponding amplitudes involving only vector bosons grow with energy when the bosons are longitudinally polarized and violate perturbative unitarity at about one $\mathrm{TeV}$, requiring either the Higgs or some new physics in the energy range accessible to the LHC in order to tame this unphysical behaviour ${ }^{1}$.

The Standard Model (SM) describes Electroweak Symmetry Breaking (EWSB) through a single complex Higgs doublet. Many alternative mechanisms of EWSB however have been explored. We will not try to summarize the different models and simply refer to the literature. We will only remark that it is conceivable that composite states are responsible for EWSB $[7,8,9,10,11,12,13,14]$. These theories typically predict the presence of new states which, if light enough, could be observed at the LHC.

The effective field theory approach $[15,16,14,17]$ is a powerful method for treating the low energy dynamics of systems with broken symmetries. It provides a systematic expansion of the full unknown Lagrangian in terms of the fields which are relevant at scales much lower than the symmetry breaking scale.

\footnotetext{
${ }^{1}$ Detailed reviews and extensive bibliographies can be found in Refs. $[1,2,3,4,5,6]$
} 
In Ref. [14] it has been pointed out that, if EWSB is triggered by a light composite Higgs which is a pseudo-Goldstone boson related to some large scale strongly interacting dynamics, the growth with energy of the vector boson scattering amplitudes typical of Higgsless models might not be completely canceled by Higgs exchange diagrams but only slowed down. This kind of models have been called Strongly Interacting Light Higgs (SILH) models. Examples which fall into this class are for instance the Holographic Higgs [12], the Little Higgs of Ref. [13] and the Littlest Higgs [9].

In SILH models the leading low energy effects are described by two parameters (one responsible for a universal modification of all Higgs couplings, and the other one for a universal modification of Higgs couplings to fermions) characterized by the ratio $v^{2} / f^{2}=\xi$, where $v$ is the Higgs vacuum expectation value and $f$ is the $\sigma$-model scale. The natural range of the $\xi$ parameter is between $\xi=0$ and $\xi=1$ which correspond respectively to the limiting cases of the Standard Model and of technicolor theories. Because of the modified Higgs couplings, longitudinal gauge-boson scattering amplitudes violate unitarity at high energy, even in the presence of a light Higgs [14].

Scattering processes among vector bosons have been scrutinized since a long time [18]. In Ref. $[19,20]$ an analysis of $\ell \nu+$ four jets and $\ell^{+} \ell^{-}+$four jets production at the LHC has been presented, with the limitation of taking into account only purely electroweak processes. Preliminary results concerning the inclusion of the $\mathcal{O}\left(\alpha_{E M}^{4} \alpha_{S}^{2}\right)$ background, which include $V V+2 j$ and top-antitop production have appeared in Ref. [21]. A preliminary analysis in the Equivalent Vector Boson Approximation of the observability of partial unitarization of longitudinal vector boson scattering in SILH models at the LHC can be found in Ref. [22]. In the last few years QCD corrections to boson-boson production via vector boson fusion [23] at the LHC have been computed and turn out to be below 10\%. Recently, VBFNLO [24] a Monte Carlo program for vector boson fusion, double and triple vector boson production at NLO QCD accuracy, limited to the leptonic decays of vector bosons, has been released. Recently, the first results for the NLO corrections to $W+4 j$ production have appeared [25]. New techniques which exploit the angular distribution of vector boson decay products to determine the ratio of longitudinal and transverse polarization have been proposed in $[26]$.

In Ref. [27] a complete parton level analysis of $\ell \nu+$ four jets production at the LHC, including all processes at order $\mathcal{O}\left(\alpha_{E M}^{6}\right), \mathcal{O}\left(\alpha_{E M}^{4} \alpha_{S}^{2}\right)$ and $\mathcal{O}\left(\alpha_{E M}^{2} \alpha_{S}^{4}\right)$ has been presented, comparing a typical SM light Higgs scenario with the Higgsless case. It was noted that the $\mathcal{O}\left(\alpha_{E M}^{2} \alpha_{S}^{4}\right) W+4 \mathrm{j}$ background is so large that the usual approach of comparing the number of events in the two scenarios at large invariant masses is useless. It was argued that the invariant mass distribution of the two central jets in the vector-vector scattering signal presents a peak corresponding to the decays of vector bosons while the background produced by $\mathcal{O}\left(\alpha_{E M}^{2} \alpha_{S}^{4}\right) W+4 j$ processes is rather flat and therefore can be measured from the sidebands and subtracted, drastically decreasing the theoretical uncertainties.

In Ref. [28] the processes $p p \rightarrow \ell^{+} \ell^{-}+4 j$ and $p p \rightarrow 3 \ell \nu+2 j$ have been studied along the lines introduced in Ref. [27]. The infinite mass Higgs scenario and the instance of SILH models described above have been compared with the light Higgs SM framework.

In this paper we concentrate on the boson boson scattering reactions which produce 
a $2 \ell 2 \nu 2 j$ final state. Because of the presence of two neutrinos the mass of the final state boson pair cannot be reconstructed. For completeness sake in the end we also discuss the $2 j 4 \ell$ channel in which the vector pair mass can obviously be measured with high accuracy but which has been left out of our previous papers because of its small cross section. These processes have been studied already in Ref. [29] where they have been described as goldplated. A potentially large background to these channels is the copious yield of high $\mathrm{p}_{T}$, isolated leptons in B-hadron production [30] which mimic the signature of the leptonic decays of $W$ bosons. A detailed experimental analysis of two same sign $W$ 's has however shown that when standard isolation criteria are applied isolated leptons from B-hadrons can be efficiently eliminated [31]. Therefore, we have reanalyzed the $2 \ell 2 \nu 2 j$ channels using complete $\mathcal{O}\left(\alpha_{E M}^{6}\right)$ and $\mathcal{O}\left(\alpha_{E M}^{4} \alpha_{S}^{2}\right)$ samples.

We have estimated the probability that, assuming that either the Higgsless scenario or the instance of SILH model we have considered is realized in Nature, the results of the measurements at the LHC yield results which are incompatible with the SM. We have first combined separately the three channels, $2 j \ell^{ \pm} \ell^{ \pm} \nu \nu, 2 j Z Z \rightarrow 2 j \ell \nu \ell \nu, 2 j W W \rightarrow 2 j \ell \nu \ell \nu$ in which the invariant mass of the final state cannot be directly measured and the four channel, $2 j 4 \ell, 4 j \ell \nu, 4 j \ell \ell$ and $2 j 3 \ell \nu$ in which it can instead be reconstructed. Finally we have combined all channels.

\section{Calculation}

Two perturbative orders contribute to the $2 \ell 2 \nu 2 j$ and $4 \ell 2 j$ signals at the LHC. The purely electroweak set of diagrams at $\mathcal{O}\left(\alpha_{E M}^{6}\right)$ is the one which includes boson boson scattering as a subprocess. In the second set of diagrams at $\mathcal{O}\left(\alpha_{E M}^{4} \alpha_{S}^{2}\right)$ no such scattering takes place: either two fermion lines exchange a gluon or a single fermion line and two external gluons are present. In contrast with the processes examined in Refs. [27, 28] where the dominating background is due to $V+4 j \mathcal{O}\left(\alpha_{E M}^{2} \alpha_{S}^{4}\right)$ processes in which only one vector boson is produced, in the present case the final states from both perturbative orders contain two vector bosons and are essentially impossible to separate. In Refs. [32, 33] it has been pointed out that $t \bar{t}+n$-jets production, $n=1,2$ can provide a significant background to vector boson scattering. Indeed, the additional jets which are present in the former processes can go undetected and mimic the signature of boson boson reactions.

Both the $\mathcal{O}\left(\alpha_{E M}^{6}\right)$ and the $\mathcal{O}\left(\alpha_{E M}^{4} \alpha_{S}^{2}\right)$ samples have been generated with PHANTOM, a dedicated tree level Monte Carlo generator which is documented in Ref. [34] while additional material can be found in Refs. [35, 36, 37]. The $t \bar{t}+n$-jets processes have been simulated with MADEVENT [38] in the Narrow Width Approximation. Both programs generate events in the Les Houches Accord File Format [39]. For the LHC we have assumed the design energy of $14 \mathrm{TeV}$. For each perturbative order we have generated a sample of five hundred thousand unweighted events. In some cases additional event samples have been generated in order to increase the final statistics of particular phase space regions.

All samples have been generated using CTEQ5L [40] parton distribution functions. 
The QCD scale has been taken as

$$
\Lambda^{2}=M_{W}^{2}+\frac{1}{6} \sum_{i=1}^{6} p_{T i}^{2}
$$

in all cases but for the reaction in which a triplet of final state particles with flavours compatible with deriving from the decay of a top or antitop quark could be found. In this case the scale has been taken as

$$
\Lambda^{2}=M_{t o p}^{2}+p_{T t o p}^{2}
$$

The analysis has been performed at parton level with no showering and hadronization. The effective Lagrangian approach to SILH models of Ref. [14] is valid for small values of $\xi$, while larger values demand a more detailed description of the particular model at hand. Such a Lagrangian leads to a modification of the Higgs couplings by a factor $1 / \sqrt{1+c_{H} \xi}$, which can be reabsorbed in a Higgs propagator modification by a factor $1 /\left(1+c_{H} \xi\right)$ in boson boson scattering studies. $c_{H}$ is a pure number of order unity $[9,12,13,14]$. For the present study we have selected the value $c_{H} \xi=1$ which we intend as a possible upper limit for the model independent lagrangian description of Ref. [14]. Both for the SM scenario and for the SILH model the Higgs mass has been fixed at $200 \mathrm{GeV}$. Since we are interested in large invariant mass vector vector scattering processes the actual value of the Higgs mass is irrelevant, provided it is appreciably smaller than the invariant mass of the vector pair.

For very large Higgs masses, all Born diagrams with Higgs propagators become completely negligible in the Unitary Gauge we work in. Therefore the no-Higgs model results for all processes coincide with those in the $M_{H} \rightarrow \infty$ limit. This framework therefore can be considered as an upper limit to SILH models and also as representative of all models in which resonances which unitarize vector vector scattering are present but too heavy to be directly detected. The no-Higgs case is also extremely useful to determine the phase space regions in which weak and strong interacting vector boson models differ the most. It is obvious that the Standard Model with an infinite mass Higgs is unphysical because of the violations of perturbative unitarity at about one $\mathrm{TeV}$. This corresponds in our complete calculation to an invariant mass of the two vector bosons of the same magnitude. These events are present at the LHC but they are highly suppressed by the effective parton luminosities, as can be clearly extracted from the plots in Refs. [27, 28] and in the following. We have studied the behaviour of unitarized models, and in particular we have compared the basic no-Higgs case with a model in which the no-Higgs amplitudes are unitarized using the $\mathrm{K}$-matrix method and with some models which contain resonances. Typically the number of expected events in the presence of resonances is much larger than for the no-Higgs framework. What is perhaps more important, it turns out that, after cuts comparable to the ones adopted in this paper, the expected number of events in the unitarized no-Higgs model is only a few percent smaller than in the non-unitarized case. Therefore we consider the possibility of distinguishing the no-Higgs case from the SM at the LHC a quite solid benchmark for the observability of unitarized models. The details of our studies of unitarized models are given in Refs. [41, 42]. 
The selection of events with jets widely separated in pseudorapidity is a well established technique for enhancing the scattering contributions at the LHC [18]. As shown in Ref. [27] a powerful tool to increase the separation between the SM predictions and those of the Higgsless scenario is provided, at large invariant masses, by the request that the vector bosons and their decay products are in the central part of the detector since the vector bosons in the Higgsless case have smaller rapidities and larger momenta than in the presence of a light Higgs.

The cuts in Tab. 1 have been applied either at generation level or as a preliminary step to any further analysis. They require containment within the active region of the detectors and minimum transverse momentum for all observed partons; a minimum mass separation is imposed for all same-family opposite-sign charged leptons and all jet pairs. Furthermore, the two jets are required to be separated by at least three $(2 j 2 \ell 2 \nu)$ or four $(2 j 4 \ell)$ units in rapidity and their combined mass is forced to be outside the electroweak vector boson mass window in order to exclude three vector boson production.

We have considered two different ranges for the mass of the lepton pair in the $2 j \ell^{+} \ell^{-} \nu \bar{\nu}$ channel. On one hand we have selected same flavour charged leptons with a mass in the interval $76 \mathrm{GeV}<M\left(\ell^{+} \ell^{-}\right)<106 \mathrm{GeV}$. In this case we have considered the lepton pair to be produced in the decay of a $Z$ boson. Requiring further a large missing transverse momentum we have produced an event sample corresponding to the $2 j Z Z \rightarrow 2 j \ell^{+} \ell^{-} \nu \bar{\nu}$ channel which will be discussed in Sect. 4 . When the mass of the lepton pair is outside the quoted range or the two oppositely charged leptons belong to different families we consider the event a candidate for the $2 j W W$ channel. Since we are interested into high invariant mass $W$ pairs, we have required $M\left(\ell^{+} \ell^{-}\right)>250 \mathrm{GeV}$ for this kind of events which will be analyzed in Sect. 5. The mass of the $W W$ system corresponds to the scale of boson boson scattering and large masses help in discriminating between the SM and other scenarios.

For both the Higgsless and SILH cases and for each channel we have computed the probability that, assuming a specific Beyond Standard Model (BSM) correctly describes nature, the result of an experimental outcome for a given luminosity has a chance of less than 5\% in the SM (PBSM@95\%CL).

For the combination of channels discussed in Sect. 7 we have also computed the $99.7 \%$ exclusion limit (PBSM@99.7\%CL).

All limits presented in the following, unless explicitly mentioned, have been computed summing over all possible combinations of first and second generation leptons, assuming an integrated luminosity of $L=200 \mathrm{fb}^{-1}$, which we intend as corresponding to one year of high luminosity combining CMS and ATLAS results.

We proceed as follows. We define the signal $S$ as the sum of the events for all $\mathcal{O}\left(\alpha_{E M}^{6}\right)$ and $\mathcal{O}\left(\alpha_{E M}^{4} \alpha_{S}^{2}\right)$ processes after all selection cuts. It might be feasible to further decrease the $\mathcal{O}\left(\alpha_{E M}^{4} \alpha_{S}^{2}\right)$ contribution with a central jet veto, but this possibility is beyond the scope of this paper. For the $2 j W W \rightarrow 2 j \ell \nu \ell \nu$ channel we take as background $B$ the expected yield of the $t \bar{t}+$ jets. $B$ and $S$ are considered as random variables representing the number of background and signal events for a possible experimental outcome. $\bar{B}$ and $\bar{S}$ are the corresponding average values which will be taken equal to the predictions of our simulation. We take into account the statistical uncertainty of $S$ assuming a standard 
Poisson distribution with average $\bar{S}$. The predicted signal cross section is also affected by theoretical uncertainties, so the parameter $\bar{S}$ is itself subject to fluctuations. The theoretical error is modeled by a flat distribution in the window $\bar{S} \pm 30 \%$ which, in our opinion, is a reasonable choice to account for both pdf's and scale uncertainties for the signal. The processes we are interested in require center of mass energies of the order of the $\mathrm{TeV}$ and therefore involve quarks with rather large longitudinal momentum fraction $x, x \approx 10^{-1} \div 10^{-2}$ at a typical scale $\Lambda$ of about $100 \mathrm{GeV}$. In this region the uncertainty due to the parton distribution functions is of the order of $5 \%[43,44]$. As already stated, QCD corrections are in the range of $10 \%$ and, as a consequence theoretical uncertainties are expected to be well within this order of magnitude.

Only statistical fluctuations have been taken into consideration in the case of $B$. This is motivated by the fact that the $t \bar{t}+$ jets background is likely to be well measured experimentally in final states in which more than two jets are detected and then extrapolated via Monte Carlo to the region of interest in this paper, so that the theoretical error on $B$ is not expected to be an issue at the time when real data analysis will be performed. In Sect. 7 we will also discuss how our results would be affected if $t \bar{t}+j e t s$ were not measured.

\begin{tabular}{|l|}
\hline$p_{T}\left(\ell^{ \pm}\right)>20 \mathrm{GeV}$ \\
\hline$\left|\eta\left(\ell^{ \pm}\right)\right|<3.0$ \\
\hline$M\left(\ell^{+} \ell^{-}\right)>20 \mathrm{GeV}$ \\
$M\left(\ell^{+} \ell^{-}\right)>250 \mathrm{GeV} \quad\left(2 j W^{+} W^{-}\right)$ \\
$76 \mathrm{GeV}<M\left(\ell^{+} \ell^{-}\right)<106 \mathrm{GeV} \quad(2 j Z Z)$ \\
\hline$p_{T}(j)>30 \mathrm{GeV}$ \\
\hline$|\eta(j)|<6.5$ \\
\hline$M(j j)>60 \mathrm{GeV}$ \\
$M(j j)<70 \mathrm{GeV} ; M(j j)>100 \mathrm{GeV}$ \\
\hline$|\Delta \eta(j j)|>3.0 \quad(2 j 2 \ell 2 \nu)$ \\
$|\Delta \eta(j j)|>4.0 \quad(2 j 4 \ell)$ \\
\hline
\end{tabular}

Table 1: Acceptance cuts.

We define the test statistics $D=$ $S+B-\bar{B}$ which reduces to $S$ in the absence of background. Having computed the probability distributions $P(D \mid S M)$ and $P(D \mid B S M)$ of $D$ in the Standard Model and in the Beyond the Standard Model under consideration, the $95 \% \mathrm{CL}$ region for the SM can be defined from the probability ratio

$$
Q(D)=\frac{P(D \mid B S M)}{P(D \mid S M)}
$$

determining a number $\alpha$ such that

$$
\int d D P(D \mid S M) \theta(\alpha-Q)=95 \% \text {. }
$$

The probability for the BSM to yield a result outside this 95\%CL region for the SM is then

$$
P B S M @ 95 \% C L=\int d D P(D \mid B S M) \theta(Q-\alpha) .
$$

A number of comments, which apply to all channels discussed in this paper, should be made. We have performed a simple cut based study, which can undoubtedly be improved upon with a more sophisticated multivariate analysis. On the other hand we have not taken 
into account experimental efficiencies and all issues related to additional hadronic activity due to showering and the underlying event. The selection cuts discussed below have been chosen in order to maximize the separation of the light Higgs case from the no-Higgs one.

In the following we will present cross sections as a function of appropriate minimum invariant masses $M_{c u t}$, typically extracted from lepton momenta. The best discrimination between the SM and the BSM schemes are generally obtained for $M_{c u t}$ values which yield production rates which are uncomfortably small, particularly because of neglected experimental uncertainties. It should however be noticed that at smaller values of $M_{\text {cut }}$ the rate is usually much larger with a modest decrease of discriminating power.

\section{The $2 j \ell^{ \pm} \ell^{\prime \pm} \nu \nu$ channel: two same--sign leptons in the final state}

This channel, which is characterized by two same sign charged leptons, possibly of different flavour, in the final state, has low EW and QCD background, for no external gluons contribute to this final state. We remark that the production of two same-sign $W$ 's has been extensively discussed in the context of Multiple Particle Interactions (MPI) [45, 46, 47], since it has the peculiarity that it can be realized in MPI at a lower perturbative order than in ordinary two parton collisions where at least two additional partons must appear in the final state. However, if two jets in the final state are required, the MPI contribution is small and concentrated in the region of small total visible energy and therefore has been neglected.

\begin{tabular}{|l|l|l|l|}
\hline $\begin{array}{l}M_{\text {cut }} \\
(\mathrm{GeV})\end{array}$ & \multicolumn{1}{|c|}{ no Higgs } & \multicolumn{1}{|c|}{ SILH } & \multicolumn{1}{c|}{$M_{H}=200 \mathrm{GeV}$} \\
\cline { 2 - 4 } & \multicolumn{1}{|c|}{$\sigma(\mathrm{fb})$} & \multicolumn{1}{|c|}{$\sigma(\mathrm{fb})$} & \multicolumn{1}{c|}{$\sigma \mathrm{fb})$} \\
\hline 200 & $3.11(2.39)$ & $2.87(2.15)$ & $2.73(2.01)$ \\
300 & $1.73(1.23)$ & $1.55(1.06)$ & $.46(.967)$ \\
400 & $1.01(.682)$ & $.892(.560)$ & $.839(.507)$ \\
500 & $.630(.407)$ & $.538(.315)$ & $.505(.283)$ \\
600 & $.400(.253)$ & $.334(.187)$ & $.311(.163)$ \\
700 & $.262(.162)$ & $.214(.114)$ & $.198(.0975)$ \\
800 & $.177(.108)$ & $.142(.0728)$ & $.130(.0613)$ \\
\hline
\end{tabular}

Table 2: Total cross section for the $\ell^{ \pm} \ell^{ \pm} \nu \nu+2 j$ channel after generation cuts, Tab. 1 . In parentheses the results for the $\mathcal{O}\left(\alpha_{E M}^{6}\right)$ sample.

The presence of two neutrinos in the final state makes it impossible to reconstruct the invariant mass of the di-boson system which corresponds to the center of mass energy of the $W W$ scattering. For $M_{c u t}$ we have resorted to a correlated observable, the di-lepton mass $M(\ell \ell)$.

The total cross section for the $2 j \ell^{ \pm} \ell^{ \pm} \nu \nu$ channel with the acceptance cuts in Tab. 1 is presented in Tab. 2 as a function of the minimum $\ell \ell$ invariant mass $M_{\text {cut }}$. In parentheses the results for the $\mathcal{O}\left(\alpha_{E M}^{6}\right)$ processes. Tab. 2 shows that the cross section for the $\mathcal{O}\left(\alpha_{E M}^{4} \alpha_{S}^{2}\right)$ processes is only about $25 \%$ to $40 \%$ of the total cross section in the Higgsless scenario already at this level. The distribution of the lepton pair mass, with acceptance cuts only, 
is presented on the left hand side of Fig. 1. The $\mathcal{O}\left(\alpha_{E M}^{4} \alpha_{S}^{2}\right)$ background is negligible at small di-lepton mass, while it becomes of the same order of magnitude of the $\mathcal{O}\left(\alpha_{E M}^{6}\right)$ contribution for $M_{\text {cut }}>500 \mathrm{GeV}$.

In the following, as already mentioned, we will consider the full sample as our signal. It is possible to improve the discriminating power of the analysis increasing the fraction of $\mathcal{O}\left(\alpha_{E M}^{6}\right)$ events in the event sample since only those are sensitive to the mechanism of EWSB. Therefore, on the generated samples we have applied the additional selection cuts shown in Tab. 3. These cuts force the two tag jets to be well separated and not central. One of the two leading jets is forbidden from having a very large transverse momentum. The two charged leptons are required to be rather central and well separated from the jets.

\begin{tabular}{|l|}
\hline$\Delta \eta(j j)>4.5$ \\
\hline $\max |\eta(j)|>2.5$ \\
\hline$|\eta(j)|>1$. \\
\hline$|\eta(\ell)|<2.5$ \\
\hline$p_{T}(\ell)>50 \mathrm{GeV}$ \\
\hline $\min p_{T}(j)<120 \mathrm{GeV}$ \\
\hline$\Delta R(\ell j)>1.5$ \\
\hline$\left|\vec{p}_{T}\left(\ell_{1}\right)-\vec{p}_{T}\left(\ell_{2}\right)\right|>150 \mathrm{GeV}$ \\
\hline $\cos \left(\delta \phi_{\ell \ell}\right)<-0.6$ \\
\hline
\end{tabular}

Table 3: Additional selection cuts for channel $2 j \ell^{ \pm} \ell^{ \pm} \nu \nu$. They are required to be well separated in the transverse plane and to have large transverse momentum. Finally, the vector difference between the lepton momenta is required to be large.
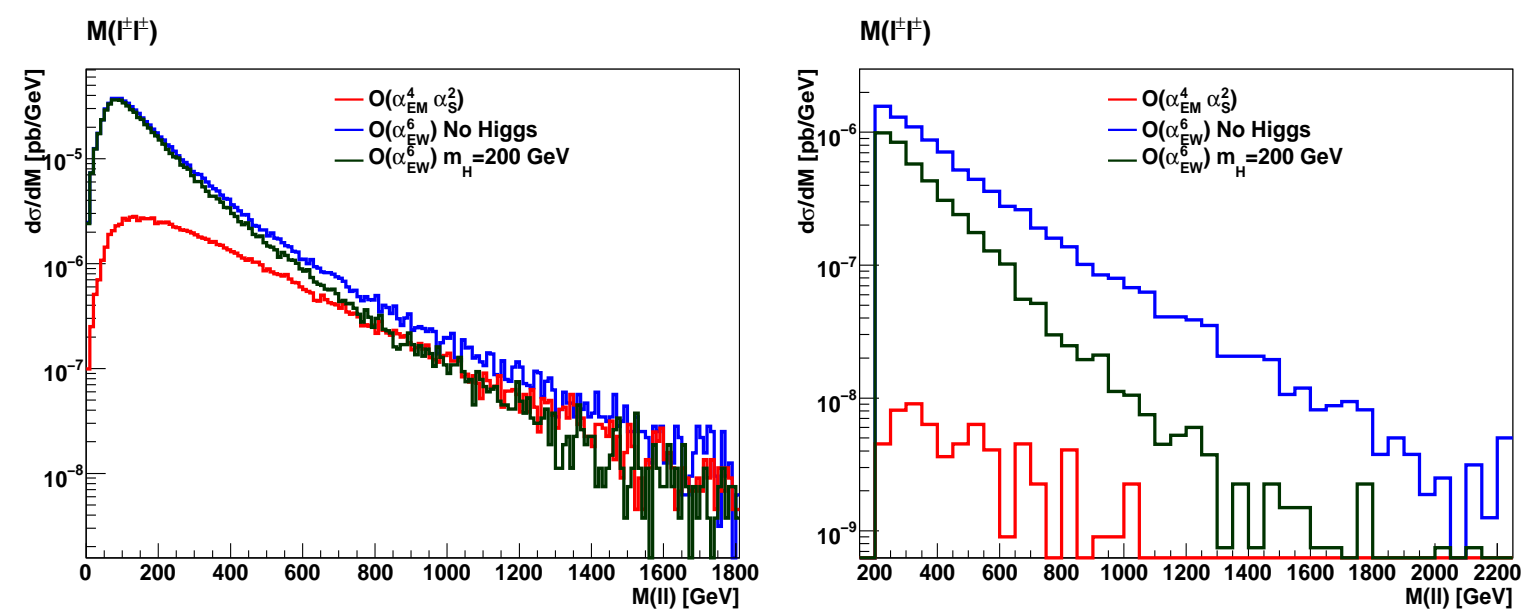

Figure 1: $M(\ell \ell)$ distribution with acceptance cuts only, Tab. 1 (left) and after all additional selection cuts, Tab. 3 (right).

The total cross section in femtobarns for the $2 j \ell^{ \pm} \ell^{ \pm} \nu \nu$ channel, with the full set of cuts in Tab. 1 and Tab. 3, as a function of the minimum invariant mass $M_{c u t}$ is shown in Tab. 4. In parentheses the results for the $\mathcal{O}\left(\alpha_{E M}^{6}\right)$ contribution, which dominate the cross section, are reported. The distribution of $M(\ell \ell)$ is presented on the right hand side of Fig. 1 which clearly demonstrates the good separation between the two scenarios obtained through the additional cuts. As expected the separation increases with increasing di-lepton invariant mass.

In Tab. 4 we also give the PBSM@95\%CL for the two BSM scenarios. The corresponding normalized frequency for the three scenarios, as a function of the number of events, is 


\begin{tabular}{|l|l|l|l|l|l|}
\hline \multirow{2}{*}{$\begin{array}{l}M_{\text {cut }} \\
(\mathrm{GeV})\end{array}$} & \multicolumn{1}{|c|}{ no Higgs } & \multicolumn{2}{c|}{ SILH } & $M_{H}=200 \mathrm{GeV}$ \\
\cline { 2 - 6 } & \multicolumn{1}{|c|}{$(\mathrm{fb})$} & PBSM & $\sigma(\mathrm{fb})$ & PBSM & $\sigma(\mathrm{fb})$ \\
\hline 200 & $.435(.431)$ & $94.9 \%$ & $.276(.273)$ & $39.1 \%$ & $.206(.203)$ \\
300 & $.290(.288)$ & $98.2 \%$ & $.166(.164)$ & $42.3 \%$ & $.114(.111)$ \\
\hline 400 & $.191(.189)$ & $98.7 \%$ & $.0977(.0958)$ & $41.2 \%$ & $.0629(.0609)$ \\
\hline 500 & $.129(.128)$ & $98.7 \%$ & $.0604(.0588)$ & $34.4 \%$ & $.0351(.0336)$ \\
600 & $.0886(.0876)$ & $97.5 \%$ & $.0385(.0375)$ & $37.1 \%$ & $.0194(.0183)$ \\
700 & $.0614(.0607)$ & $96.6 \%$ & $.0262(.0254)$ & $42.3 \%$ & $.0112(.0105)$ \\
800 & $.0438(.0432)$ & $91.1 \%$ & $.0184(.0178)$ & $31.2 \%$ & $.00701(.00640)$ \\
\hline
\end{tabular}

Table 4: Total cross section for the $\ell^{ \pm} \ell^{ \pm} \nu \nu+2 j$ channel in femtobarns, with the full set of cuts in Tab. 1 and Tab. 3, as a function of the minimum dilepton invariant mass $M_{c u t}$ for the $\ell^{ \pm} \ell^{ \pm}$ system. In parentheses the results for the $\mathcal{O}\left(\alpha_{E M}^{6}\right)$ sample. We also show the PBSM probabilities. The result for $M_{c u t}=400 \mathrm{GeV}$ which provides the best discrimination between the Higgsless and light Higgs scenario is highlighted.

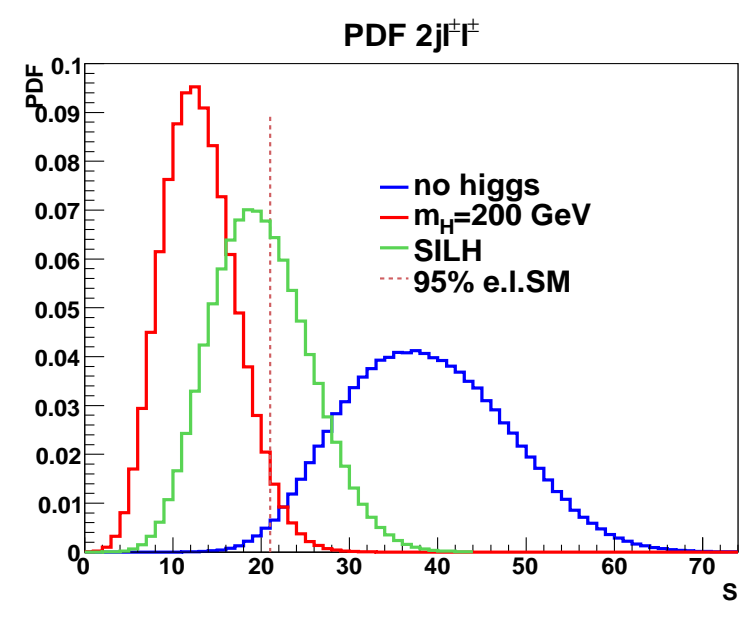

Figure 2: Probability distribution for no-Higgs, SILH and SM cases for the $2 j \ell^{ \pm} \ell^{ \pm} \nu \nu$ channel. The vertical line indicates the $95 \% \mathrm{CL}$ for the SM. $M_{\text {cut }}=400 \mathrm{GeV}$.

reported in Fig. 2 for $M_{c u t}=400 \mathrm{GeV}$. The red curve refers to the probability distribution for a Higgs of $200 \mathrm{GeV}$ while the green one refers to the SILH model and the blue one to the no-Higgs case. The dotted vertical line in the plot marks the $95 \%$ exclusion limit for the SM predictions.

The probability of an experiment to find a result incompatible with the SM at 95\%CL, assuming that the Higgsless model is realized in Nature, is of the order of $99 \%$ for $M_{c u t}=$ $400 \mathrm{GeV}$ and decreases to about $90 \%$ for $M_{c u t}=800 \mathrm{GeV}$. Because of the absence of large backgrounds this channel has a discriminating power which is in fact quite high. The corresponding probabilities for the SILH model vary between $30 \%$ and $40 \%$.

About 40(20) events are predicted for the Higgsless(SILH) scenario assuming our standard luminosity of $L=200 \mathrm{fb}^{-1}$ and $M_{c u t}=400 \mathrm{GeV}$, which provides the best discrimination between the Higgsless scenario and the SM. 


\section{The $2 j Z Z \rightarrow 2 j \ell^{+} \ell^{-} \nu \bar{\nu}$ channel: two opposite-sign same-flavour leptons in the final state}

This channel has been separated from the $2 j W W \rightarrow 2 j \ell^{+} \ell^{-} \nu \bar{\nu}$ case using the di-lepton mass. If $\left|M(\ell \ell)-M_{Z}\right|<15 \mathrm{GeV}$, the event is considered as produced by a $Z Z$ intermediate state. Since the mass of the final state $Z Z$ system cannot be fully reconstructed we estimate the center of mass energy of the vector boson scattering from the transverse mass:

$$
M_{T}^{2}(Z Z)=\left[\sqrt{M_{Z}^{2}+p_{T}^{2}(\ell \ell)}+\sqrt{M_{Z}^{2}+p_{T m i s s}^{2}}\right]^{2}-\left|\overrightarrow{p_{T}}(\ell \ell)+\vec{p}_{T m i s s}\right|^{2}
$$

The total cross section for the $2 j Z Z \rightarrow 2 j \ell^{+} \ell^{-} \nu \bar{\nu}$ channel with the acceptance cuts in Tab. 1 is presented in Tab. 5 as a function of the minimum $M_{c u t}=M_{T}(Z Z)$. In parentheses the results for the $\mathcal{O}\left(\alpha_{E M}^{6}\right)$ processes.

The $M_{T}(Z Z)$ distribution, with acceptance cuts only, is presented on the left hand side of Fig. 3. The QCD background is much larger than in the channels discussed in Sect. 3. The contribution from top pair production is large, particularly at small transverse masses, even though we are requiring a lepton pair with an invariant mass in the neighborhood of the $Z$ mass. This contribution rapidly fades at large $M_{T}(Z Z)$ where the QCD processes without top are dominating. Moreover, since no requirement of large missing transverse momentum has been imposed, additional backgrounds at small transverse masses are generated by $2 j Z \rightarrow 2 j \ell^{+} \ell^{-}$production. We have not included this background contribution in our study since large $p_{\text {Tmiss }}$ is demanded in our final analysis and this additional contribution is completely eliminated.

\begin{tabular}{|l|l|l|l|}
\hline \multirow{2}{*}{$\begin{array}{l}M_{\text {cut }} \\
(\mathrm{GeV})\end{array}$} & \multicolumn{1}{|c|}{ no Higgs } & \multicolumn{1}{|c|}{ SILH } & \multicolumn{1}{c|}{$M_{H}=200 \mathrm{GeV}$} \\
\cline { 2 - 4 } & \multicolumn{1}{|c|}{$\sigma(\mathrm{fb})$} & \multicolumn{1}{c|}{$\sigma(\mathrm{fb})$} & \multicolumn{1}{c|}{$\sigma \mathrm{fb})$} \\
\hline 300 & $1.84(.607)$ & $1.73(.494)$ & $.544(.187)$ \\
400 & $.675(.319)$ & $.578(.222)$ & $.262(.0962)$ \\
500 & $.363(.197)$ & $.288(.122)$ & $.140(.0515)$ \\
600 & $.223(.134)$ & $.161(.0727)$ & $.0781(.0300)$ \\
700 & $.143(.0952)$ & $.0947(.0466)$ & $.0426(.0186)$ \\
800 & $.0926(.0686)$ & $.0553(.0313)$ & $.0251(.0120)$ \\
900 & $.0646(.0515)$ & $.0341(.0210)$ & \\
\hline
\end{tabular}

Table 5: Total cross section for the $(Z Z) \ell^{+} \ell^{-} \nu \nu+2 j$ channel after generation cuts, Tab. 1. In parentheses the results for the $\mathcal{O}\left(\alpha_{E M}^{6}\right)$ sample.

In order to sharpen the separation between the Standard Model results and those from alternative symmetry breaking scenarios we impose the additional cuts in Tab. 6 .

The total cross section in femtobarns for the $2 j Z Z \rightarrow 2 j \ell^{+} \ell^{-} \nu \bar{\nu}$ channel, with the full set of cuts in Tab. 1 and Tab. 6, as a function of the minimum $Z Z$ transverse mass $M_{c u t}$ is shown in Tab. 7. In parentheses the results for the $\mathcal{O}\left(\alpha_{E M}^{6}\right)$ contribution. The PBSM probabilities are also given. 

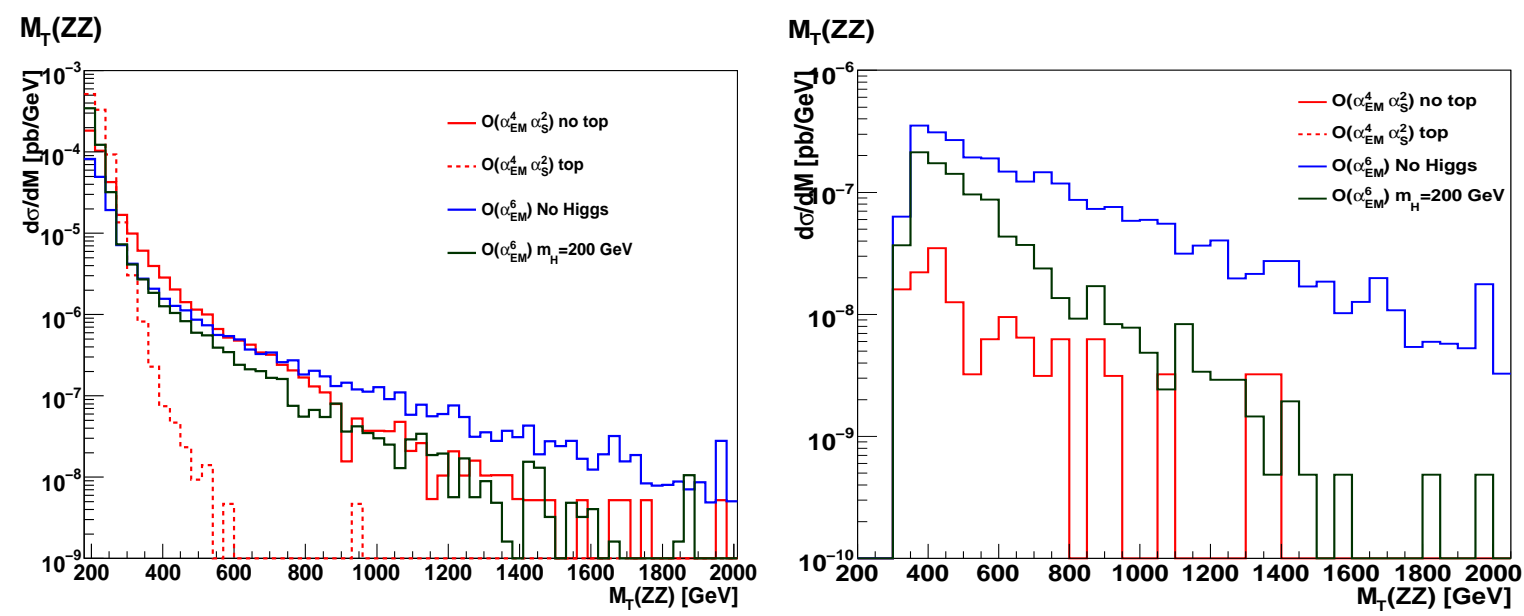

Figure 3: ZZ transverse mass distribution with initial cuts, Tab. 1 (left) and adding extra cuts, Tab. 6 (right).

\begin{tabular}{|l|l|l|l|l|l|}
\hline \multirow{2}{*}{$\begin{array}{l}M_{\text {cut }} \\
(\mathrm{GeV})\end{array}$} & \multicolumn{1}{|c|}{ no Higgs } & \multicolumn{2}{c|}{ SILH } & $M_{H}=200 \mathrm{GeV}$ \\
\cline { 2 - 6 } & \multicolumn{1}{|c|}{$(\mathrm{fb})$} & PBSM & $\sigma(\mathrm{fb})$ & PBSM & $\sigma(\mathrm{fb})$ \\
\hline 300 & $.143(.136)$ & $94.6 \%$ & $.0770(.0700)$ & $31.5 \%$ & $.0540(.0470)$ \\
400 & $.120(.115)$ & $96.1 \%$ & $.0614(.0564)$ & $36.2 \%$ & $.0396(.0345)$ \\
500 & $.0887(.0860)$ & $97.5 \%$ & $.0396(.0369)$ & $39.8 \%$ & $.0214(.0187)$ \\
\hline 600 & $.0691(.0668)$ & $98.4 \%$ & $.0268(.0246)$ & $44.3 \%$ & $.0118(.00957)$ \\
\hline 700 & $.0547(.0533)$ & $97.0 \%$ & $.0186(.0171)$ & $32.0 \%$ & $.00697(.00555)$ \\
800 & $.0410(.0401)$ & $94.6 \%$ & $.0145(.0136)$ & $33.2 \%$ & $.00463(.00368)$ \\
900 & $.0327(.0321)$ & $94.3 \%$ & $.00991(.00927)$ & $31.9 \%$ & $.00300(.00236)$ \\
\hline
\end{tabular}

Table 7: Total cross section for the $(Z Z) \ell^{+} \ell^{-} \nu \nu+2 j$ channel in femtobarns, with the full set of cuts in Tab. 1 and Tab. 6, as a function of the minimum transverse mass $M_{T}(Z Z)_{\text {cut }}$. In parentheses the results for the $\mathcal{O}\left(\alpha_{E M}^{6}\right)$ sample. The PBSM@95\%CL are also shown.

The $M_{T}(Z Z)$ distribution, with the full set of cuts, is presented on the right hand side of Fig. 3 . The $\mathcal{O}\left(\alpha_{E M}^{4} \alpha_{S}^{2}\right)$ background has been sharply reduced while increasing the separation between the Higgsless and light Higgs scenarios. The top related background has been totally suppressed.

The probability distribution for the three scenarios is reported in Fig. 4 for $M_{T}(Z Z)_{c u t}=600 \mathrm{GeV}$. The red curve refers to a Higgs of $200 \mathrm{GeV}$ while the green one refers to the SILH model and the blue

\begin{tabular}{|l|}
\hline$\Delta \eta(j j)>4.5$ \\
\hline$M(j j)>800 \mathrm{GeV}$ \\
\hline$\Delta \eta(\ell j)>1.3$ \\
\hline$p_{T m i s s}>120 \mathrm{GeV}$ \\
\hline$\left|\vec{p}_{T}\left(\ell^{+} \ell^{-}\right)-\vec{p}_{T}^{\text {miss }}\right|>290 \mathrm{GeV}$ \\
\hline$p_{T}\left(\ell^{+} \ell^{-}\right)>120 \mathrm{GeV}$ \\
\hline$|\eta(j)|>1.9$ \\
\hline
\end{tabular}

Table 6: Selection cuts for channel $(Z Z) \ell^{+} \ell^{-} \nu \nu+2 j$. one to the no-Higgs case. The dotted vertical line in the plot marks the $95 \%$ exclusion limit for the SM predictions. The probability of an experiment to find a result incompatible with the SM at $95 \% \mathrm{CL}$, assuming that the Hig- 
PDF 2jZZ

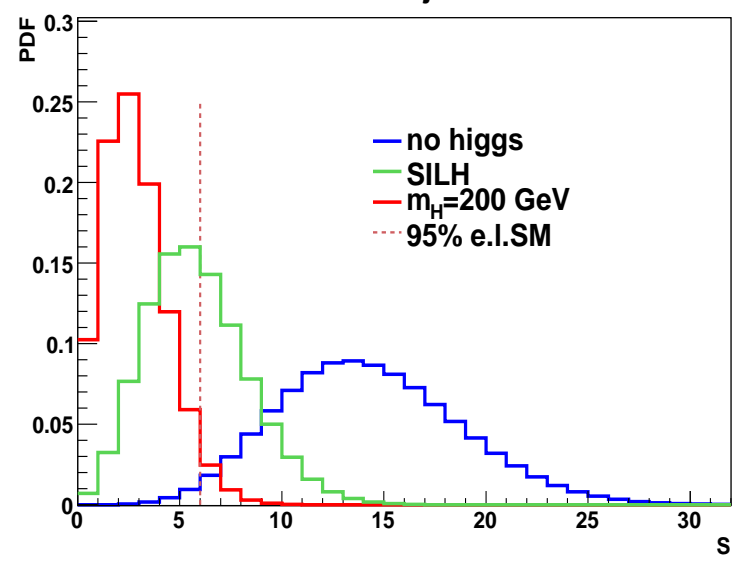

Figure 4: Probability distribution for the no-Higgs, SILH and SM cases for the $2 j Z Z \rightarrow 2 j \ell^{+} \ell^{-} \nu \bar{\nu}$ channel. The vertical line indicates the $95 \% \mathrm{CL}$ for the SM. $M_{c u t}=600 \mathrm{GeV}$.

gsless model is realized in Nature, is of the order of $98 \%$ for $M_{c u t}=600 \mathrm{GeV}$ and does not vary significantly over the range considered in Tab. 7 . The corresponding probabilities for the SILH model vary between $30 \%$ and $45 \%$.

\section{The $2 j W^{+} W^{-} \rightarrow 2 j \ell^{+} \ell^{\prime-} \nu \bar{\nu}$ channel: two opposite-sign leptons in the final state}

The total cross section for the $2 j W^{+} W^{-} \rightarrow 2 j \ell^{+} \ell^{-} \nu \bar{\nu}$ channel, with the acceptance cuts in Tab. 1 , is shown in Tab. 8 as a function of the minimum $\ell \ell$ invariant mass. As usual, in parentheses we show the results for the $\mathcal{O}\left(\alpha_{E M}^{6}\right)$ processes. The cross sections for $t \bar{t} j$ and $t \bar{t} j j$ production are presented separately. We have required exactly two jets in the acceptance region. This however is not sufficient to guarantee a well defined cross section because two $b$ quarks are produced in the leptonic decay of the tops. Therefore we have further required that one $b$ for $t \bar{t} j$ events and both $b$ 's for $t \bar{t} j j$ ones remain undetected. We consider a $b$-quark detected if $\left|\eta_{b}\right|<5$ and $p_{T b}>30 \mathrm{GeV}$. As a consequence the partons produced in association with the $t \bar{t}$ pair are forced to be visible and the corresponding cross section is finite. The phase space regions which are excluded by these constraints are eliminated by the cut on the mass of all lepton-jet pairs discussed below.

This process has the largest production rate among all channels considered in this paper, however the QCD background is much larger than the electroweak part.

The $M(\ell \ell)$ distribution, with acceptance cuts only, is presented on the left hand side of Fig. 5. $t \bar{t}$ production is very important at this level, and the usual way to suppress it, by requiring $M(W j)$ out of the top nominal mass window, is not applicable because of the impossibility to reconstruct the $W$ mass. Instead we require the mass of all lepton-jet pairs to be larger than the top mass.

The relatively high signal rate and the large background allow and require harder cuts than in all previous cases. The additional selection requirements are shown in Tab. 9. The 


\begin{tabular}{|c|c|c|c|c|c|}
\hline $\begin{array}{c}M_{\text {cut }} \\
(\mathrm{GeV})\end{array}$ & no Higgs & SILH & $M_{H}=200 \mathrm{GeV}$ & $t \bar{t} j$ & $t \bar{t} j j$ \\
\cline { 2 - 6 } & $\sigma(\mathrm{fb})$ & $\sigma(\mathrm{fb})$ & $\sigma(\mathrm{fb})$ & $\sigma(\mathrm{fb})$ & $\sigma(\mathrm{fb})$ \\
\hline 300 & $70.0(4.65)$ & $69.7(4.42)$ & $69.7(4.35)$ & 39.7 & 2.59 \\
400 & $29.7(2.32)$ & $29.5(2.11)$ & $29.5(2.12)$ & 16.4 & 1.22 \\
500 & $13.5(1.24)$ & $13.4(1.14)$ & $13.4(1.13)$ & 7.21 & .516 \\
600 & $6.69(.713)$ & $6.62(.643)$ & $6.60(.627)$ & 3.13 & .237 \\
700 & $3.55(.440)$ & $3.49(.376)$ & $3.47(.362)$ & 1.45 & .139 \\
800 & $1.92(.274)$ & $1.88(.236)$ & $1.87(.225)$ & .849 & .0737 \\
\hline
\end{tabular}

Table 8: Total cross section for the $\left(W^{+} W^{-}\right) \ell^{+} \ell^{-} \nu \nu+2 j$ channel after initial cuts, Tab. 1 in function of the minimum $\ell \ell$ invariant mass, $M(\ell \ell)$. In parentheses the results for the $\mathcal{O}\left(\alpha_{E M}^{6}\right)$ sample.

constraint on the lepton-jet mass is quite effective in reducing the background due to top pair production. The $t \bar{t}$ and $t \bar{t} j$ contributions are essentially eliminated and the two light partons in $t \bar{t} j j$ production are forced to be tagged. However this cut reduces significantly the boson scattering signal and furthermore it increases the relative contribution of $t \bar{t} j j$.

The total cross section in femtobarns for the $\left(W^{+} W^{-}\right) \ell^{+} \ell^{-} \nu \nu+2 j$ channel, with the full set of cuts in Tab. 1 and Tab. 9, as a function of the minimum di-lepton invariant mass $M_{\text {cut }}$ is shown in Tab. 10. In parentheses the results for the $\mathcal{O}\left(\alpha_{E M}^{6}\right)$ contribution. The PBSM probabilities are also presented. As mentioned in Sect. 2 only the statistical uncertainty has been taken into account for the $t \bar{t} j j$ background. The requirement that both $b$-quarks have a transverse momentum smaller than $30 \mathrm{GeV}$ or a rapidity in modulus larger than 5 units is quite stringent, in fact the cross section for $t \bar{t} j j$ requiring both $b$ 's to produce visible jets, not necessarily identified as $b$-jets, is at least an order of magnitude larger than the results presented in Tab. 10.

\begin{tabular}{|l|}
\hline$M(j j)>1000 \mathrm{GeV}$ \\
\hline$\Delta \eta(j j)>4.8$ \\
\hline$|\eta(\ell)|<2.00$ \\
\hline$p_{T}(\ell)>40 \mathrm{GeV}$ \\
\hline$m a x|\eta(j)|>2.5$ \\
\hline$|\eta(j)|>1.3$ \\
\hline$E(j)>180 \mathrm{GeV}$ \\
\hline$\Delta \eta(\ell j)>0.8$ \\
$(\operatorname{and} \Delta R(\ell j)>1)$ \\
\hline$M(\ell j)>180 \mathrm{GeV}$ \\
\hline$\left|\vec{p}_{T}\left(\ell^{+}\right)-\vec{p}_{T}\left(\ell^{-}\right)\right|>220 \mathrm{GeV}$ \\
\hline $\cos \left(\delta \phi_{\ell \ell}\right)<-0.6$ \\
\hline
\end{tabular}

Table 9: Additional selection cuts for the $2 j W^{+} W^{-} \rightarrow 2 j \ell^{+} \ell^{-} \nu \nu$ channel. Therefore, we believe the $t \bar{t} j j$ will be measured in the complementary region and extrapolated to the signal domain with small uncertainty. In any case, since the hadronic activity is expected to be much higher in $t \bar{t}+$ jets events than in boson boson scattering ones, a more accurate assessment of this background would require complete showering and hadronization.

We will come back to the impact on our results in case the $t \bar{t} j j$ could not be measured in Sect. 7. For the time being we only report that the PBSM@95\%CL reported in Tab. 10 for $M_{\text {cut }}=600 \mathrm{GeV}$ would change from $85 \%$ to $78 \%$ for the no Higgs case and from $27 \%$ to $22 \%$ for the SILH model.

The di-lepton mass distribution, with the full set of cuts, is presented on the right hand side of Fig. 5. The $\mathcal{O}\left(\alpha_{E M}^{4} \alpha_{S}^{2}\right)$ background is now very small while the separation 

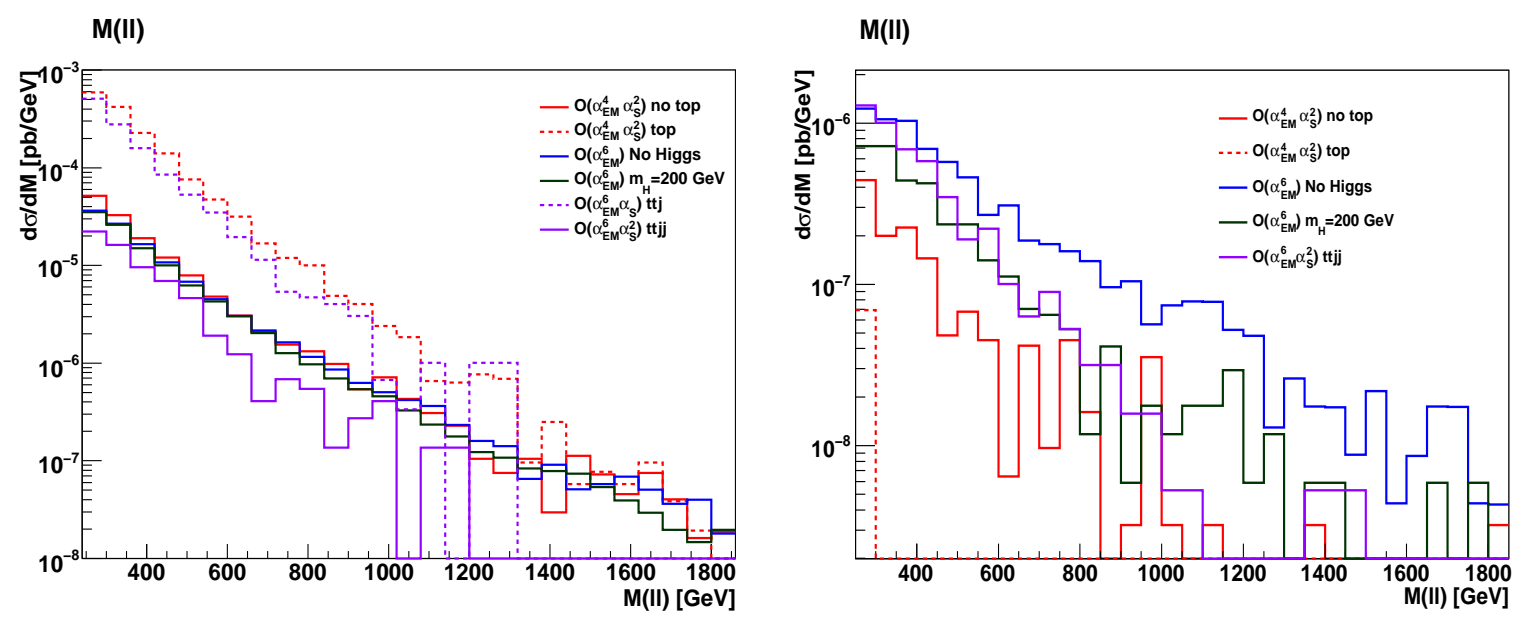

Figure 5: Di-lepton mass distribution with initial cuts, Tab. 1 (left) and adding extra cuts, Tab. 9 (right).

\begin{tabular}{|c|c|c|c|c|c|c|}
\hline$M_{\text {cut }}$ & \multicolumn{2}{|c|}{ no Higgs } & \multicolumn{2}{c|}{ SILH } & $M_{H}=200$ & $t \bar{t} j j$ \\
\cline { 2 - 7 }$(\mathrm{GeV})$ & $\sigma(\mathrm{fb})$ & PBSM & $\sigma(\mathrm{fb})$ & PBSM & $\sigma(\mathrm{fb})$ & $\sigma(\mathrm{fb})$ \\
\hline 300 & $.337(.292)$ & $79.58 \%$ & $.224(.179)$ & $22.69 \%$ & $.179(.134)$ & .173 \\
400 & $.212(.188)$ & $80.74 \%$ & $.131(.107)$ & $20.89 \%$ & $.100(.0765)$ & .0890 \\
500 & $.139(.125)$ & $82.83 \%$ & $.0841(.0700)$ & $26.35 \%$ & $.0577(.0435)$ & .0407 \\
\hline 600 & $.0968(.0883)$ & $85.03 \%$ & $.0533(.0448)$ & $26.56 \%$ & $.0332(.0247)$ & .0215 \\
\hline 700 & $.0696(.0635)$ & $80.55 \%$ & $.0353(.0292)$ & $20.63 \%$ & $.0217(.0156)$ & .0138 \\
\hline
\end{tabular}

Table 10: Total cross section for the $2 j W^{+} W^{-} \rightarrow 2 j \ell^{+} \ell^{-} \nu \nu$ channel in femtobarns, with the full set of cuts in Tab. 1 and Tab. 9, as a function of the minimum di-lepton invariant mass $M(\ell \ell)_{c u t}$. In parentheses the results for the $\mathcal{O}\left(\alpha_{E M}^{6}\right)$ sample. The PBSM@95\%CL are given in the third and fifth column.

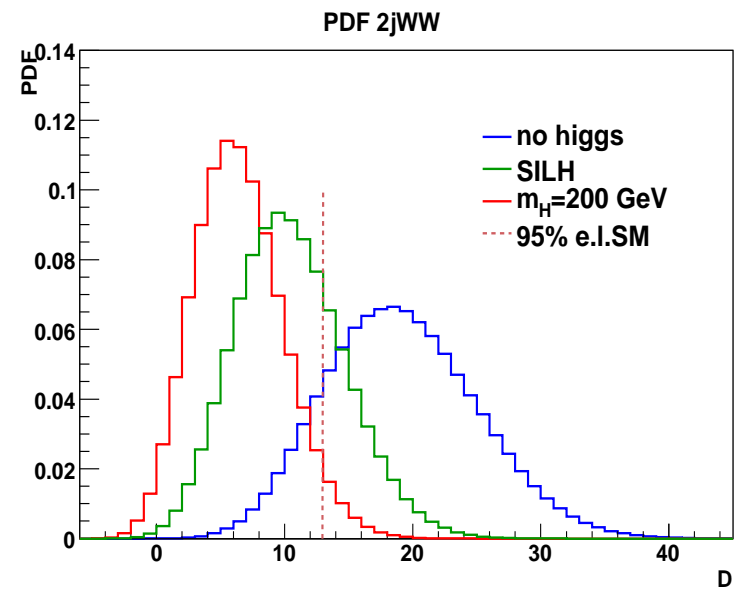

Figure 6: Probability distribution for the no-Higgs, SILH and SM cases for the $2 j W^{+} W^{-} \rightarrow$ $2 j \ell^{+} \ell^{-} \nu \bar{\nu}$ channel. The vertical line indicates the $95 \% \mathrm{CL}$ for the SM. $M_{c u t}=600 \mathrm{GeV}$. 
between the Higgsless and light Higgs scenarios is clearly visible.

The probability distribution for the three scenarios is reported in Fig. 6 for $M(l l)_{c u t}=$ $600 \mathrm{GeV}$. The probability of an experiment to find a result incompatible with the SM at 95\%CL, assuming that the Higgsless model is realized in Nature, is between 80 and $85 \%$ for $300 \mathrm{GeV}<M_{\text {cut }}<700 \mathrm{GeV}$. For the SILH model the corresponding probabilities lie between 20 and $26 \%$.

\section{The $2 j 4 \ell$ channel}

Contrary to all reaction discussed above, in the $2 j 4 \ell$ channel the mass of the final state vector boson pair can be directly measured to a high precision. It is presented here for completeness despite its small rate and statistical discriminating power.

The QCD contribution is small already at generation level. However, for a luminosity of $L=200 \mathrm{fb}^{-1}$ the difference between the number of events expected for an infinite mass Higgs and a light one is of the order the statistical uncertainty for the $\mathcal{O}\left(\alpha_{E M}^{4} \alpha_{S}^{2}\right)$ contribution and no meaningful separation between the two cases can be obtained. Only a minimum set of additional cuts can be applied in order to have at least a handful of events for $L=200 \mathrm{fb}^{-1}$. This channel could clearly profit from

\begin{tabular}{|l|}
\hline$M(j j)>800 \mathrm{GeV}$ \\
\hline$p_{T}(Z)>100 \mathrm{GeV}$ \\
\hline$\Delta R(Z j)>1$ \\
\hline $\cos \left(\delta \phi_{Z Z}\right)<-0.4$ \\
\hline
\end{tabular}

Table 11: Additional selection cuts for the $2 j 4 \ell$ channel. higher luminosities. The additional selection cuts are shown in Tab. 11.

\begin{tabular}{|l|l|l|l|l|l|}
\hline \multirow{2}{*}{$\begin{array}{l}M_{\text {cut }} \\
(\mathrm{GeV})\end{array}$} & \multicolumn{2}{|c|}{ no Higgs } & \multicolumn{2}{c|}{ SILH } & $M_{H}=200 \mathrm{GeV}$ \\
\cline { 2 - 6 } & \multicolumn{1}{|c|}{$(\mathrm{ab})$} & PBSM & \multicolumn{1}{c|}{$\sigma(\mathrm{ab})$} & PBSM & $\sigma(\mathrm{ab})$ \\
\hline 300 & $51.8(41.6)$ & $35.6 \%$ & $36.1(26.0)$ & $8.4 \%$ & $31.6(21.5)$ \\
400 & $44.7(36.7)$ & $40.7 \%$ & $30.1(22.1)$ & $10.3 \%$ & $25.5(17.5)$ \\
\hline 500 & $35.6(30.1)$ & $41.8 \%$ & $22.8(17.3)$ & $10.5 \%$ & $18.4(12.9)$ \\
\hline 600 & $28.2(24.2)$ & $34.1 \%$ & $17.2(13.2)$ & $7.0 \%$ & $13.5(9.45)$ \\
700 & $22.2(19.5)$ & $29.3 \%$ & $12.8(10.0)$ & $5.3 \%$ & $9.64(6.93)$ \\
800 & $17.8(15.8)$ & $29.1 \%$ & $9.79(7.82)$ & $5.4 \%$ & $7.09(5.12)$ \\
900 & $14.0(12.6)$ & $31.0 \%$ & $7.38(6.05)$ & $6.7 \%$ & $5.19(3.87)$ \\
\hline
\end{tabular}

Table 12: Total cross section for the $4 \ell+2 j$ channel in attobarns, with the full set of cuts in Tab. 1 and Tab. 11, as a function of the minimum invariant mass $M_{c u t}$ for the $4 \ell$ system. In parentheses the results for the $\mathcal{O}\left(\alpha_{E M}^{6}\right)$ sample. Also shown are the PBSM probabilities.

The cross section after these extra cuts is presented in Tab. 12 as a function of the minimum $Z Z$ mass. In parentheses the results for the $\mathcal{O}\left(\alpha_{E M}^{6}\right)$ sample. We also show the PBSM@95\%CL for the standard $L=200 \mathrm{fb}^{-1}$ luminosity.

The probability distribution of the discriminant $S$ for the three scenarios is reported in Fig. 7 for $M_{\text {cut }}=500 \mathrm{GeV}$ with the full set of cuts in Tab. 1 and Tab. 11.

As reported in Tab. 12, the probability of an experiment to find a result incompatible with the SM at 95\%CL, assuming that the Higgsless model is realized in Nature, is of the 


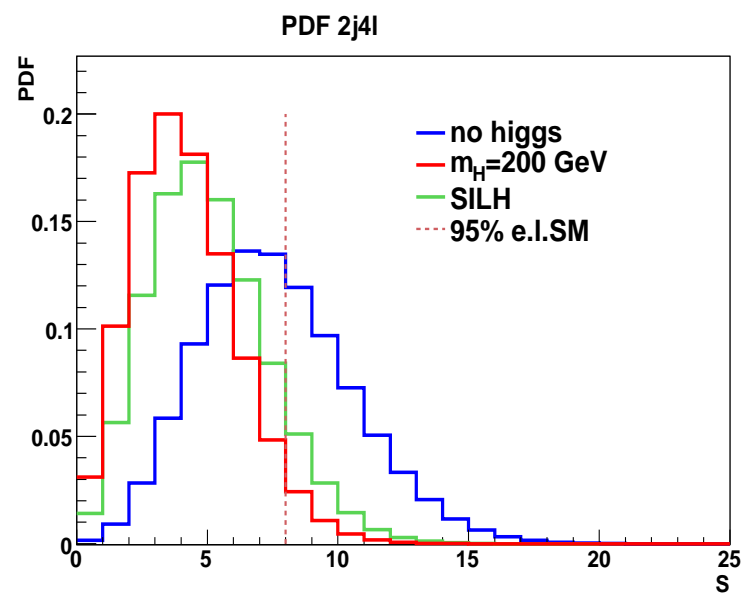

Figure 7: Probability distribution for the no-Higgs, SILH and SM cases for the $2 j 4 \ell$ channel. The vertical line indicates the $95 \%$ CL for the SM. $M_{c u t}=500 \mathrm{GeV}$.

order of $42 \%$ for $M_{\text {cut }}=500 \mathrm{GeV}$ and decreases to about $30 \%$ for $M_{\text {cut }}=700 \mathrm{GeV}$. For the SILH model the PBSM@95\%CL is only about $10 \%$ at most, for $M_{\text {cut }}=500 \mathrm{GeV}$.

For $L=200 \mathrm{fb}^{-1}$ and summing over all final states, the expected total rates are of the order of $4 \div 8$ events for the Higgsless case.

\section{Combining all channels}

In this section we derive the probability that, assuming that either the Higgsless scenario or the instance of SILH model we have considered is realized in Nature, the results of the measurements of the seven channels $2 j \ell^{ \pm} \ell^{ \pm} \nu \nu, 2 j Z Z \rightarrow 2 j \ell \nu \ell \nu, 2 j W W \rightarrow 2 j \ell \nu \ell \nu, 2 j 4 \ell$, $4 j \ell \nu, 4 j \ell \ell$ and $2 j 3 \ell \nu$ at the $\mathrm{LHC}$ yield results which are outside the $95 \%$ probability region for the SM.

Tab. 13 shows the number of expected events for the reactions in which the $V V$ mass can be reconstructed. The data for $4 j \ell \nu$ production are taken from Ref. [27] while those for $4 j \ell^{+} \ell^{-}$and $2 j \ell^{+} \ell^{-} \ell^{\prime} \nu$ are from Ref. [28] to which we refer for more details. Tab. 14 instead shows the number of expected events for the channels in which direct reconstruction of the vector boson pair mass is impossible. In both instances the assumed integrated luminosity is $200 \mathrm{fb}^{-1}$. In each case the prediction corresponds to the value of $M_{\text {cut }}$ which gives the best PBSM@95\%CL. These values are highlighted in Tables 4, 7, 10 and 12 for the reactions described in detail in this paper. For the remaining channels $M_{c u t}$ has been set to $600 \mathrm{GeV}$.

For a given number of events for each channel $k_{1}, k_{2}, \ldots, k_{n}$, with corresponding mean values $\lambda_{1}, \lambda_{2}, \ldots, \lambda_{n}$, which we will refer to collectively as $\vec{k}$ and $\vec{\lambda}$, the standard likelihood ratio in Eq.(2.3) can be expressed as $Q\left(\vec{k} ; \vec{\lambda}_{B S M}, \vec{\lambda}_{S M}\right)=P\left(\vec{k}, \vec{\lambda}_{B S M}\right) / P\left(\vec{k}, \vec{\lambda}_{S M}\right)$

The procedure we have employed so far and in Ref. [28] to evaluate the PBSM becomes cumbersome when too many channels have to be considered and the dimensionality of the integrals in Eqs. $(2.4,2.5)$ becomes large. Therefore for the combination of all results we 
have resorted to the variable $-2 \ln Q$. From the one dimensional probability distribution of $-2 \ln Q$ the $95 \%$ CL and $99.7 \%$ CL limits for the SM can be easily determined.

In the following we will first combine separately the first three channels, in which the invariant mass of the $V V$ pair cannot be reconstructed, and the last four, in which the $V V$ mass can be directly measured. Later we will proceed to a full combination.

\begin{tabular}{|l|c|c|c|c|c|}
\hline & $\mathrm{S}$ (noHiggs) & $\mathrm{S}(\mathrm{SILH})$ & $\mathrm{S}\left(m_{H}=200 \mathrm{GeV}\right)$ & $\mathrm{B} \mathcal{O}\left(\alpha_{E M}^{2} \alpha_{S}^{4}\right)$ & $t \bar{t} j j$ \\
\hline $4 j \ell \nu$ & 473.6 & 281.6 & 210.4 & 1956. & 92.6 \\
\hline $4 j \ell^{+} \ell^{-}$ & 61.6 & 30.4 & 19.38 & 220. & - \\
\hline $2 j \ell^{+} \ell^{-} \ell^{\prime} \nu$ & 10.8 & 5.4 & 3.4 & - & - \\
\hline $2 j \ell^{+} \ell^{-} \ell^{+} \ell^{-}$ & 7.12 & 4.56 & 3.68 & - & - \\
\hline
\end{tabular}

Table 13: Number of events expected for $L=200 \mathrm{fb}^{-1}$ for the channels in which the $V V$ mass can be reconstructed. The cuts for the first three reactions are described in [27] and [28]. The cuts for the $2 j 4 \ell$ channel are discussed in Sect. 6. $M_{\text {cut }}$ is chosen in such a way that the best PBSM@95\%CL for each channel is obtained.

\begin{tabular}{|l|c|c|c|c|}
\hline & S(noHiggs) & S(SILH) & S $\left(m_{H}=200 \mathrm{GeV}\right)$ & $t \bar{t} j j$ \\
\hline $2 j \ell^{ \pm} \ell^{ \pm} \nu \nu$ & 38.2 & 19.54 & 12.58 & - \\
\hline $2 j Z Z \rightarrow \ell^{+} \ell^{-} \nu \nu$ & 13.82 & 5.36 & 2.36 & - \\
\hline $2 j W^{+} W^{-} \rightarrow \ell^{+} \ell^{-} \nu \nu$ & 19.36 & 10.66 & 6.64 & 4.3 \\
\hline
\end{tabular}

Table 14: Number of events expected for $L=200 \mathrm{fb}^{-1}$ for the channels in which the $V V$ mass cannot be reconstructed. $\quad M_{c u t}$ is chosen in such a way that the best PBSM@95\%CL for each channel is obtained.

The probability $P(\vec{k}, \vec{\lambda})$ depends on the correlations between channels. In our simplified approach in which only statistical and theoretical errors are accounted for, only the uncertainties which are related to theory are correlated. Statistical errors in each channel are independent.

As a first step, we assume each channel to be subject to an independent theoretical error which is implemented by smearing the mean value for each channel separately and then combining the smeared channels. The corresponding probability, for the simple case in which the $\mathcal{O}\left(\alpha_{E M}^{2} \alpha_{S}^{4}\right)$ background is absent,is given by:

$$
P_{U}(\vec{k} ; \vec{\lambda})=\prod_{i} \int d x_{i} \rho\left(x_{i}\right) \mathcal{P}\left(k_{i},\left(1+x_{i}\right) \lambda_{i}\right)
$$

where $\mathcal{P}(k, \lambda)$ is the standard Poisson distribution with mean $\lambda$ and

$$
\rho(x)=\left\{\begin{aligned}
\frac{1}{2 \times 0.3} & \text { if }|x|<0.3 \\
0 & \text { otherwise }
\end{aligned}\right.
$$

models the (flat) theoretical uncertainty.

Combining separately the two set of channels we obtain the probabilities to exclude the SM at 95\%CL and at 99.7\%CL shown in Tab. 15. 


\begin{tabular}{|l|c|c|c|c|}
\hline \multicolumn{5}{|c|}{ uncorrelated error } \\
\hline & non-reconstructable & \multicolumn{2}{c|}{ reconstructable } \\
\hline & NOH & SILH & NOH & SILH \\
\hline $95 \%$ CL & $>99.99 \%$ & $76.24 \%$ & $99.96 \%$ & $52.81 \%$ \\
\hline $99.7 \%$ CL & $99.98 \%$ & $40.34 \%$ & $99.37 \%$ & $18.61 \%$ \\
\hline
\end{tabular}

Table 15: Probability to exclude the SM with different confidence levels, for different strong alternative scenarios combining the three non-reconstructable channels $\left(2 j \ell^{ \pm} \ell^{ \pm} \nu \nu, Z Z \rightarrow 2 j \ell \nu \ell \nu\right.$ and $W W \rightarrow 2 j \ell \nu \ell \nu)$, and the four reconstructable ones $(4 j \ell \nu, 4 j \ell \ell, 2 j 3 \ell \nu$ and $2 j 4 \ell)$. Theoretical errors are assumed to be uncorrelated as in Eq.(7.1).

\begin{tabular}{|l|c|c|c|c|}
\hline \multicolumn{5}{|c|}{ strongly-correlated error } \\
\hline & non-reconstructable & \multicolumn{2}{c|}{ reconstructable } \\
\hline & NOH & SILH & NOH & SILH \\
\hline $95 \%$ CL & $99.99 \%$ & $66.05 \%$ & $99.34 \%$ & $44.07 \%$ \\
\hline $99.7 \%$ CL & $99.66 \%$ & $34.33 \%$ & $94.24 \%$ & $16.07 \%$ \\
\hline
\end{tabular}

Table 16: Probability to exclude the SM with different confidence levels, for different strong alternative scenarios combining the three non-reconstructable channels $\left(2 j \ell^{ \pm} \ell^{ \pm} \nu \nu, Z Z \rightarrow 2 j \ell \nu \ell \nu\right.$

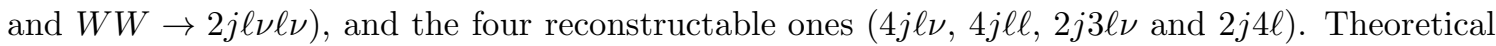
errors are assumed to be fully correlated as in Eq.(7.3).

The hypothesis that the theoretical errors in each channel are independent may underestimate the actual uncertainty. The dominant production mechanisms are the same for all channels and therefore it is likely that both pdf and scale uncertainties are fully correlated between channels. Assuming this to be the case for the total theoretical uncertainty, the average values of each channel which enter the combination must be shifted by a common factor and the corresponding probability $P_{C}$ can be expressed as

$$
P_{C}(\vec{k} ; \vec{\lambda})=\int d x \rho(x) \prod_{i} \mathcal{P}\left(k_{i},(1+x) \lambda_{i}\right) .
$$

The PBSM@95\%CL and PBSM@99.7\%CL in case of complete correlation between theoretical errors are shown in Tab. 16. Comparing Tab. 16 with Tab. 15, it is clear that dropping the hypothesis of independent theoretical errors degrades little the overall probability. Within the present theoretical framework it remains certain that the no-Higgs case would be distinguished from the SM case. In the SILH model the PBSM@95\%CL drops from $76 \%$ to $66 \%$ for the non-reconstructable channels and from $53 \%$ to $44 \%$ for the reconstructable ones.

The non-reconstructable channels presented here, independently of the detailed treatment of the theoretical uncertainty, provide a better discrimination between the SM and the BSM scenarios, despite their low rates, than those in which the invariant mass of the boson pair can be measured. This is clearly related to the absence of huge QCD backgrounds, which are instead present in the $V+4 j$ channels. The statistical uncertainties of these background are large and spoil the significance of the corresponding channels even 
when the backgrounds are assumed to be measured from the sidebands of the weak boson peak in the mass distribution of the two central jets and then subtracted as proposed in Refs. [27, 28].
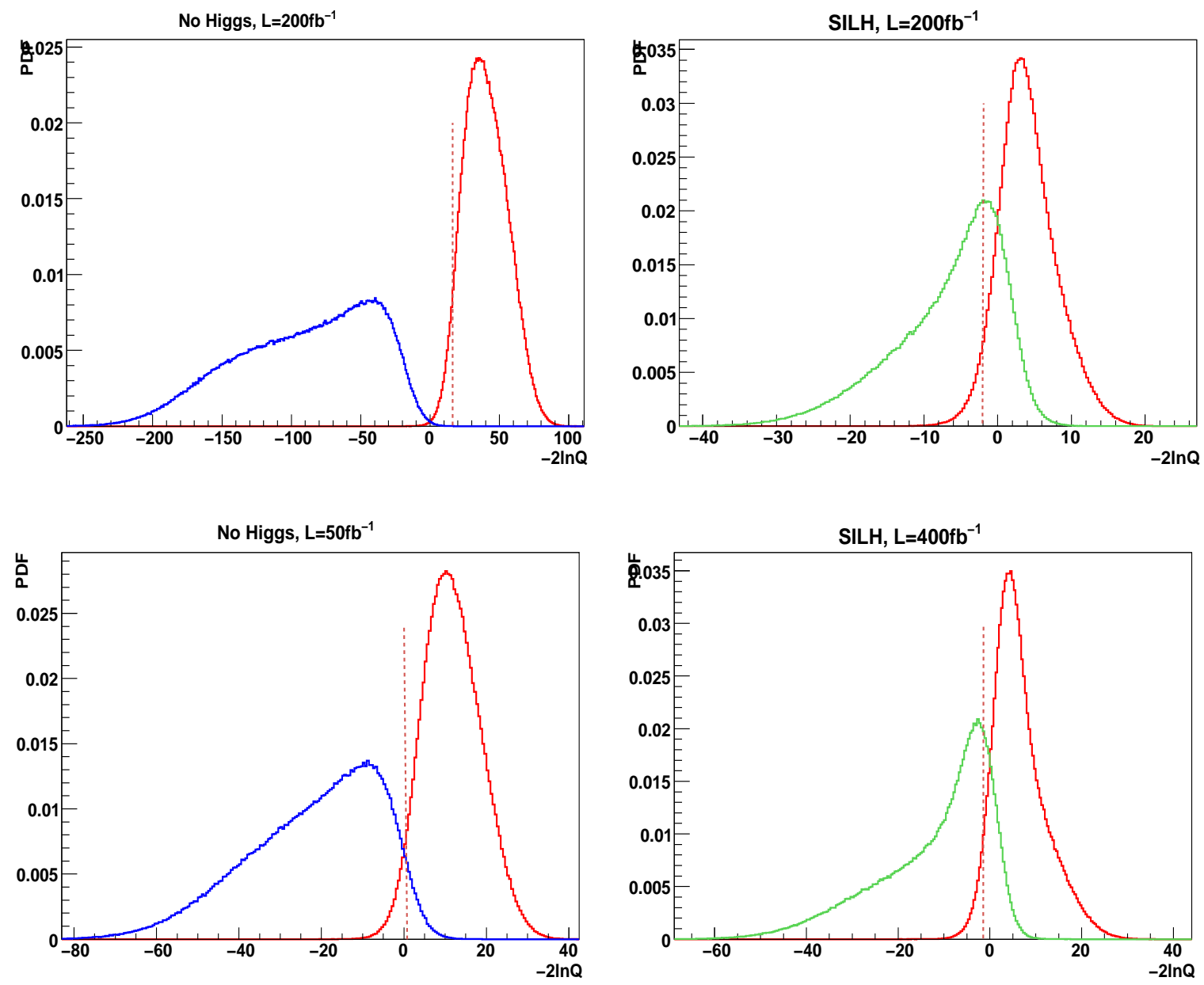

Figure 8: Combination of all the seven channels using $-2 \ln Q$ for the no-Higgs and SILH cases using strongly correlated theoretical errors. In the bottom plots an integrated luminosity of $L=$ $50 \mathrm{fb}^{-1}$ is assumed for the no-Higgs scenario and of $L=400 \mathrm{fb}^{-1}$ for the SILH case. For the no-Higgs analysis at $L=50 \mathrm{fb}^{-1}$ we have used the lowest mass cut for each channel in order to increase the number of events at this luminosity. Hence in this case $M_{c u t}=200,300,300,300 \mathrm{GeV}$ for the $2 j \ell^{ \pm} \ell^{ \pm} \nu \nu, Z Z \rightarrow 2 j \ell \nu \ell \nu, W W \rightarrow 2 j \ell \nu \ell \nu$ and $2 j 4 \ell$ channel respectively. For the remaining channels $M_{\text {cut }}$ has been kept at $600 \mathrm{GeV}$.

The distribution of $-2 \ln Q$, for the combination of all seven channels, in the Higgsless and SILH scenarios with our standard luminosity are shown in the top row of Fig. 8. In the bottom row of Fig. 8 we present the distributions for the Higgsless case with a luminosity of $50 \mathrm{fb}^{-1}$ and for the SILH model with $L=400 \mathrm{fb}^{-1}$. In all cases theoretical errors are treated as fully correlated. For the no-Higgs case at $L=50 \mathrm{fb}^{-1}$ we have adopted the lowest invariant mass cut reported in Tables 4, 7, 10 and 12 for the reactions discussed in this paper, in order to increase the number of events at this luminosity. Explicitly, 


\begin{tabular}{|l|c|c|c|c|}
\hline \multicolumn{5}{|c|}{ total combination } \\
\hline & NOH & SILH & NOH $\left(L=50 \mathrm{fb}^{-1}\right)$ & SILH $\left(L=400 \mathrm{fb}^{-1}\right)$ \\
\hline $95 \% \mathrm{CL}$ & $>99.99 \%$ & $69.32 \%$ & $96.31 \%$ & $80.82 \%$ \\
\hline $99.7 \% \mathrm{CL}$ & $99.96 \%$ & $41.67 \%$ & $83.64 \%$ & $57.16 \%$ \\
\hline
\end{tabular}

Table 17: Statistical combination of all seven channels. Also shown are the results using an integrated luminosity of $L=50 \mathrm{fb}^{-1}$ for the no-Higgs and of $L=400 \mathrm{fb}^{-1}$ for the SILH scenario. For the no-Higgs analysis we have used the lowest mass cut for each channel in order to increase the number of events at this luminosity.

$M_{c u t}=200,300,300,300 \mathrm{GeV}$ for the $2 j \ell^{ \pm} \ell^{ \pm} \nu \nu, Z Z \rightarrow 2 j \ell \nu \ell \nu, W W \rightarrow 2 j \ell \nu \ell \nu$ and $2 j 4 \ell$ channel respectively. For the remaining channels $M_{c u t}$ has been kept at $600 \mathrm{GeV}$. The corresponding PBSM@95\%CL and PBSM@99.7\%CL are given in Tab. 17. Assuming an integrated luminosity of $200 \mathrm{fb}^{-1}$ the PBSM@95\%CL for the SILH case is about $69 \%$ which increases to $80 \%$ if the luminosity is doubled. The corresponding figures for the PBSM@99.7\%CL are $42 \%$ and 57\% respectively. The probability to distinguish at 95\% CL the no-Higgs case from the light Higgs picture with a reduced luminosity of $L=50 \mathrm{fb}^{-1}$ remains above $95 \%$.

\begin{tabular}{|l|c|c|c|c|c|c|c|c|c|}
\hline Channel & \multicolumn{3}{|c|}{ no Higgs } & \multicolumn{3}{c|}{ SILH } & SM & $t \bar{t} j j$ & B \\
\hline & $\sigma(\mathrm{fb})$ & $t \bar{t} j j \subset S$ & $t \bar{t} j j=B$ & $\sigma(\mathrm{fb})$ & $t \bar{t} j j \subset S$ & $t \bar{t} j j=B$ & $\sigma(\mathrm{fb})$ & $\sigma(\mathrm{fb})$ & $\sigma(\mathrm{fb})$ \\
\hline $2 j \ell \nu \ell \nu$ & .0968 & $77.9 \%$ & $85.0 \%$ & .0533 & $22.0 \%$ & $26.6 \%$ & .0332 & .0215 & - \\
\hline $4 j \ell \nu$ & 2.36 & $90.1 \%$ & $96.2 \%$ & 1.41 & $30.9 \%$ & $35.2 \%$ & 1.05 & .463 & 9.78 \\
\hline
\end{tabular}

Table 18: PBSM@95\%CL for $200 \mathrm{fb}^{-1}$ with $t \bar{t} j j$ as as part of the signal $(t \bar{t} j j \subset S)$ and $t \bar{t} j j$ as a background $(t \bar{t} j j=B)$. We also give the total cross section for the $2 j W^{+} W^{-} \rightarrow 2 j \ell^{+} \ell^{-} \nu \nu$ and $4 j l \nu$ channels with the full set of cuts and $M_{c u t}=600 \mathrm{GeV}$.

\begin{tabular}{|c|c|c|c|c|c|c|}
\hline & \multicolumn{2}{|c|}{ non-reconstructable } & \multicolumn{2}{c|}{ reconstructable } & \multicolumn{2}{c|}{ all channels } \\
\hline scenario & $t \bar{t} j j=B$ & $t \bar{t} j j \subset S$ & $t \bar{t} j j=B$ & $t \bar{t} j j \subset S$ & $t \bar{t} j j=B$ & $t \bar{t} j j \subset S$ \\
\hline SILH & $64.93 \%$ & $63.25 \%$ & $44.07 \%$ & $40.46 \%$ & $68.38 \%$ & $67.47 \%$ \\
\hline NOH & $99.98 \%$ & $99.97 \%$ & $99.34 \%$ & $98.27 \%$ & $>99.99 \%$ & $>99.99 \%$ \\
\hline
\end{tabular}

Table 19: PBSM@95\%CL combining the non-reconstructable channels, the reconstructable ones and finally all channels with $t \bar{t} j j$ as as part of the signal $(t \bar{t} j j \subset S)$ and $t \bar{t} j j$ as a background $(t \bar{t} j j=B)$. For the $2 j W^{+} W^{-} \rightarrow 2 j \ell^{+} \ell^{-} \nu \nu$ process $M_{c u t}=500 \mathrm{GeV}$ has been used.

Before stating our conclusions we return for completeness to the possible modification to our results if the $t \bar{t} j j$ turns out not to be measurable, even though as discussed previously we do not regard this prospect as probable. In this case the contribution from $t \bar{t} j j$ production should be considered as part of the signal S and therefore subject to theoretical uncertainties in addition to the statistical ones. For simplicity we have assumed the same range of variation for this process as for all others involved. We refer to this possibility as $t \bar{t} j j$ as signal $(t \bar{t} j j \subset S)$ while the framework in which $t \bar{t} j j$ production is considered as a measured and extrapolated background is described as $t \bar{t} j j$ as background $(t \bar{t} j j=B)$. In 
Tab. 18 we present the cross sections and the PBSM@95\%CL for the two channels which are affected by the $t \bar{t} j j$ background, namely $2 j W^{+} W^{-}$and $4 j W$. In Tab. 19 we compare the two results for the different combinations of channels. While there is a noticeable decrease of the PBSM for the individual reactions in Tab. 18, the overall combinations are hardly affected.

These results suggest that the no-Higgs scenario can be disproved with a rather modest luminosity. This implies that any model which predicts vector vector scattering rates larger than those in the no-Higgs case can be disproved or verified with the same luminosity.

Our conclusions for the SILH framework are less optimistic. Clearly a substantial increase in luminosity and a combination of the results obtained by ATLAS and CMS are highly desirable. Furthermore, it should be recalled that the particular instance of SILH model we have discussed is a rather extreme case and that for smaller values of $c_{H} \xi$ results even closer to the SM ones are expected.

\section{Conclusions}

We have examined in detail at parton level the processes $2 \ell 2 \nu 2 j$ and $4 \ell 2 j, \ell=\mu, e$ including all irreducible backgrounds contributing to these six parton final states. We have considered three scenarios: a light Higgs SM framework with $M_{H}=200 \mathrm{GeV}$, one instance of the SILH models and an infinite mass Higgs scenario in order to determine whether the two BSM models can be distinguished from the SM at the LHC using boson-boson scattering. Because of the absence of large QCD backgrounds, the non-reconstructable channels presented here provide a better discrimination between the SM and the BSM scenarios, despite their low rates, than those in which the invariant mass of the boson pair can be measured.

The results for the channels discussed above have been combined with those obtained in Ref. [27] for $4 j \ell \nu$ production and those obtained in Ref. [28] for $4 j \ell^{+} \ell^{-}$and $2 j 3 \ell \nu$. We have estimated the total probability, in the two BSM scenarios, of finding a result outside the $95 \%$ probability range in the Standard Model. This probability turns out to be essentially $100 \%$ for the Higgsless case and $69 \%$ for the SILH model. These probabilities

correspond to an integrated luminosity of $L=200 \mathrm{fb}^{-1}$ and to the sum of all electron and muon channels.

\section{Acknowledgments}

A.B. wishes to thank the Dep. of Theoretical Physics of Torino University for support. This work has been supported by MIUR under contract 2006020509004 and by the European Community's Marie-Curie Research Training Network under contract MRTN-CT2006-035505 Tools and Precision Calculations for Physics Discoveries at Colliders 


\section{References}

[1] Proceedings of the Large Hadron Collider Workshop, Aachen 1990, CERN Report 90-10, G. Jarlskog and D. Rein (eds.).

[2] A. Djouadi, The Anatomy of Electro-Weak Symmetry Breaking. Tome I: The Higgs in the Standard Model, [arXiv:hep-ph/0503172].

[3] ATLAS Collaboration, Detector and Physics Performance Technical Design Report, Vols. 1 and 2, CERN-LHCC-99-14 and CERN-LHCC-99-15.

[4] K.A. Assamagan, M. Narain, A. Nikitenko, M. Spira, D. Zeppenfeld (conv.) et al., Report of the Higgs Working Group, Proceedings of the Les Houches Workshop on "Physics at TeV Colliders", 2003, [arXiv:hep-ph/0406152].

[5] CMS Collaboration, Technical Design Report, Vols. 1 and 2, CERN/LHCC 2006-001 and CERN/LHCC 2006-021.

[6] M.S. Chanowitz, Strong WW scattering at the end of the 90's: theory and experimental prospects. In Zuoz 1998, Hidden symmetries and Higgs phenomena 81-109. [arXiv:hep-ph/9812215]

[7] D. B. Kaplan and H. Georgi, Phys. Lett. B 136 (1984) 183.

[8] N. Arkani-Hamed, A. G. Cohen and H. Georgi, Phys. Lett. B 513 (2001) 232.

[9] N. Arkani-Hamed, A. G. Cohen, E. Katz and A.E. Nelson, JHEP 0207, 034 (2002), [arXiv:hep-ph/0206021].

[10] N. S. Manton, Nucl. Phys. B 158 (1979) 141; Y. Hosotani, Annals Phys. 190 (1989) 233.

[11] C. Csaki, C. Grojean and H. Murayama, Phys. Rev. D 67 (2003) 085012; C. A. Scrucca, M. Serone and L. Silvestrini, Nucl. Phys. B 669 (2003) 128.

[12] K. Agashe, R. Contino and A. Pomarol, Nucl. Phys. B 719 (2005) 165.

[13] S. Chang, JHEP 0312, 057 (2003), [arXiv:hep-ph/0306034].

[14] G.F. Giudice, C. Grojean, A. Pomarol, R. Rattazzi, JHEP 0706:045,2007, [arXiv:hep-ph/0703164].

[15] T. Appelquist and C.W. Bernard, Phys. Rev. D22 (1980) 200; A.C. Longhitano, Phys. Rev. D22 (1980) 1166; A.C. Longhitano, Nucl. Phys. B188 (1981) 118;T. Appelquist and G.H. Wu, Phys. Rev. D48 (1993) 3235(1993) [hep-ph/9304240].

[16] R. Contino, T. Kramer, M. Son and R. Sundrum, JHEP 0705 (2007) 074.

[17] R. Barbieri, B. Bellazzini, V.S. Rychkov, A. Varagnolo, Phys.Rev.D76:115008,2007, arXiv:0706.0432 [hep-ph].

[18] M.J. Duncan, G.L. Kane and W.W. Repko, Nucl. Phys. B272 (1986) 517; D.A. Dicus and R. Vega, Phys. Rev. Lett. 57 (1986) 1110; R.N. Cahn, S.D. Ellis, R. Kleiss and W.J. Stirling, Phys. Rev. D35 (1987) 1626; V. Barger, T. Han and R. Phillips, Phys. Rev. D37 (1988) 2005 and D36 (1987) 295; R. Kleiss and J. Stirling, Phys. Lett. $200 B$ (1988) 193; V. Barger et al., Phys. Rev. D42 (1990) 3052; V. Barger et al., Phys. Rev. D44 (1991) 1426; V. Barger et al., Phys. Rev. D46 (1992) 2028; D. Froideveaux, in Ref. [1] Vol II, p. 444; M. H. Seymour, in Ref. [1] Vol II, p. 557; U. Baur and E.W.N. Glover, Phys. Lett. B252 (1990) 683; D. Dicus, J. Gunion and R. Vega, Phys. Lett. B258 (1991) 475; D. Dicus, J. 
Gunion, L. Orr and R. Vega, Nucl. Phys. B377 (1991) 31; J. Bagger et al.,Phys. Rev. D49 (1994) 1246;V. Barger, R. Phillips and D. Zeppenfeld, Phys. Lett. B346 (1995) 106;

J. Bagger et al.,Phys. Rev. D52 (1995) 3878;K. Iordanidis and D. Zeppenfeld, Phys. Rev.

D57 (1998) 3072; R. Rainwater and D. Zeppenfeld, Phys. Rev. D60 (1999) 113004; erratum ibid D61 (2000) 099901.

[19] E. Accomando, A. Ballestrero, S. Bolognesi, E. Maina and C. Mariotti, JHEP 0603 (2006) 093 [arXiv:hep-ph/0512219].

[20] E. Accomando, A. Ballestrero, A. Belhouari and E. Maina, Phys. Rev. D 75 (2007) 113006 [arXiv:hep-ph/0603167].

[21] G. Bevilacqua, in F. Ambroglini et al., Proceedings of the Workshop on Monte Carlo's, Physics and Simulations at the LHC PART II, Frascati, Italy.

[22] K. Cheung, C. Chiang and T. Yuan, Phys. Rev.D78(2008)051701, arXiv:0803.2661 [hep-ph].

[23] B. Jäger, C. Oleari and D. Zeppenfeld, JHEP 0607 (2006) 015,[hep-ph/0603177]; B. Jäger, C. Oleari and D. Zeppenfeld, Phys.Rev.D73:113006,2006, [hep-ph/0604200]; G. Bozzi, B. Jäger, C. Oleari and D. Zeppenfeld, Phys.Rev.D75:073004,2007, [hep-ph/0701105]; B. Jäger, C. Oleari and D. Zeppenfeld, Phys.Rev.D80:034022,2009, arXiv:0907.0580 [hep-ph].

[24] K. Arnold et al., Comput.Phys.Commun.180:1661,2009, arXiv:0811.4559 [hep-ph].

[25] C.F. Berger et al., arXiv:1009.2338 [hep-ph].

[26] T. Han, D. Krohn, L.T. Wang and W. Zhu, JHEP 1003:082,2010, arXiv:0911.3656 [hep-ph].

[27] A. Ballestrero, G. Bevilacqua and E. Maina JHEP 05 (2009) 015, arXiv:0812.5084 [hep-ph].

[28] A. Ballestrero, G. Bevilacqua, D. Buarque Franzosi and E. Maina JHEP 11 (2009) 126, arXiv:0909.3838 [hep-ph].

[29] J. Bagger et al.,Phys. Rev. D49 (1994) 1246;J. Bagger et al.,Phys. Rev. D52 (1995) 3878.

[30] Z. Sullivan and E.L. Berger, Phys. Rev. 74 (2006) 033008, Z. Sullivan and E.L. Berger, Phys. Rev. 78 (2008) 034030.

[31] Bo Zhu et al., arXiv:1010.5848 [hep-ex].

[32] O.J.P. Eboli, M.C. Gonzalez-Garcia and J.K. Mizukoshi, Phys. Rev. D74 (2006) 073005, [hep-ph/0606118].

[33] C. Englert, B. Jager, M. Worek and D. Zeppenfeld, Phys. Rev. D80 (2009) 035027, [arXiv:0810.4861].

[34] A. Ballestrero, A. Belhouari, G. Bevilacqua, V. Kashkan and E. Maina, Comp. Phys. Commun. 180 (2009) 401, arXiv:0801.3359 [hep-ph].

[35] E. Accomando, A. Ballestrero, E. Maina, JHEP 0507 (2005) 016, [arXiv:hep-ph/0504009].

[36] A. Ballestrero and E. Maina, Phys. Lett. B350 (1995) 225, [arXiv:hep-ph/9403244].

[37] A. Ballestrero, PHACT 1.0 - Program for Helicity Amplitudes Calculations with Tau matrices' [arXiv:hep-ph/9911318] in Proceedings of the 14th International Workshop on High Energy Physics and Quantum Field Theory (QFTHEP 99), B.B. Levchenko and V.I. Savrin eds. (SINP MSU Moscow), pg. 303. 
[38] F. Maltoni, T. Stelzer, JHEP 0302 (2003) 027; T. Stelzer and W. F. Long, Comput. Phys. Commun. 81 (1994) 357;

J. Alwall et al., JHEP 0709:028,2007, arXiv:0706.2334;

H. Murayama, I. Watanabe and K. Hagiwara, KEK-91-11.

[39] J. Alwall et al., A Standard format for Les Houches event files. Written within the framework of the MC4LHC-06 workshop: Monte Carlos for the LHC: A Workshop on the Tools for LHC Event Simulation (MC4LHC), Geneva, Switzerland, 17-16 Jul 2005, Comp. Phys. Commun. 176 (2007) 300, [arXiv:hep-ph/0609017].

[40] CTEQ Coll.(H.L. Lai et al.) Eur. Phys. J. C12 (2000) 375.

[41] D. Buarque Franzosi, Ph.D Thesis.

[42] A. Ballestrero, D. Buarque Franzosi, E. Maina and L. Oggero, Vector Boson Scattering at the LHC: counting experiments for unitarized models in a full six fermion approach, in preparation.

[43] A.D. Martin, R.G. Roberts, W.J. Stirling and R.S. Thorne, Eur. Phys. J. C28 (2003) 455, [hep-ph/0211080].

[44] A.D. Martin, R.G. Roberts, W.J. Stirling and R.S. Thorne, Eur. Phys. J. C35 (2004) 325, [hep-ph/0308087].

[45] A. Kulesza and W.J. Stirling, Phys. Lett. B475 (2000) 168, [hep-ph/9912232].

[46] E. Maina, JHEP 09 (2009) 081, arXiv:0909.1586 [hep-ph].

[47] J.R. Gaunt, C. Kom, A. Kulesza and W.J. Stirling, arXiv:1003.3953. 